\title{
Ablación cardiaca auricular: estrategias guiadas por el mapeo de electrogramas
}

\section{Catheter Ablation for Atrial Fibrillation: Electrogram-Guided Methods}

\author{
A. Orozco-Duque 1, 2, C. Morillo3, C. Tobón ${ }^{4}$, J. P. Ugarte ${ }^{5}$, J. Bustamante ${ }^{2}$ \\ 'Grupo de Investigación e Innovación Biomédica, Instituto Tecnológico Metropolitano, Medellín, Colombia \\ ²Grupo de Dinámica Cardiovascular, Centro de Bioingeniería, Universidad Pontificia Bolivariana, Medellín, Colombia \\ ${ }^{3}$ Cardiology Division, Libin Cardiovascular Institute, University of Calgary, Calgary, Canadá \\ ${ }^{4}$ MATBIOM, Facultad de Ciencias Básicas, Universidad de Medellín, Medellín, Colombia \\ ${ }^{5}$ GIMSC, Facultad de Ingenierías, Universidad de San Buenaventura, Medellín, Colombia
}

\section{RESUMEN}

La ablación por radiofrecuencia se ha constituido como la técnica más utilizada para el tratamiento intervencionista de la fibrilación auricular. El aislamiento eléctrico de venas pulmonares se ha convertido en el procedimiento convencional, principalmente en pacientes con fibrilación auricular paroxística. Sin embargo, la tasa de éxito mediante esta técnica en pacientes con fibrilación auricular persistente es alrededor del 50\%. Aunque se han propuesto diversas estrategias para guiar al electrofisiólogo en los procedimientos de ablación, estudios recientes muestran que la generación de líneas de ablación adicionales guiadas anatómicamente o mediante mapeo de electrogramas complejos fragmentados, no mejora la tasa de éxito del procedimiento convencional de aislamiento de venas pulmonares. En esta revisión, se consideran las limitaciones que representan los métodos de mapeo electrofisiológicos actuales, las nuevas estrategias de evaluación de los electrogramas y los métodos de procesamiento de señales que se ven propuestos en el futuro más inmediato, para guiar los procedimientos de ablación particularmente en pacientes con fibrilación auricular persistente.

PALABRAS CLAVE: fibrilación auricular; ablación por catéter; electrogramas auriculares 


\section{ABSTRACT}

Radiofrequency catheter ablation has evolved into an effective treatment option for drug-resistant patients with atrial fibrillation. Electrical isolation of the pulmonary veins has become the standard ablation strategy mainly in patients with paroxysmal atrial fibrillation. However, the success rate of pulmonary veins isolation is about $50 \%$ in patients with persistent atrial fibrillation. Although different strategies to guide the electrophysiologist in ablation procedures have been proposed. Recent studies show that the generation of additional ablation lines guided anatomically or by fragmented complex electrograms mapping does not improve the success rate of the conventional pulmonary veins isolation procedure. In this review, we describe the limitations of current electrophysiological mapping methods, the new electrogram evaluation strategies and the signal processing methods that are proposed in the immediate future, to guide ablation procedures, particularly in patients with atrial fibrillation persistent.

KEYwORDS: atrial fibrillation; catheter ablation; atrial electrograms

\section{Correspondencia}

DESTINATARIO: Andrés Orozco Duque

INSTITUCIÓN: Instituto Tecnológico Metropolitano

DIRECCIÓN: Carrera 65 \#98 A-75, Medellín,

Antioquia, Colombia

CORREO ELECTRÓNICO: andresorozco@itm.edu.co

\section{Fecha de recepción:}

4 de diciembre de 2017

Fecha de aceptación:

14 de junio de 2018 


\section{INTRODUCCIÓN}

La fibrilación auricular (FA) es una de las taquiarritmias más comunes: afecta alrededor del $2 \%$ de la población general y su incidencia está aumentando ${ }^{[1]}$. La FA está asociada con anormalidades hemodinámicas y eventos tromboembólicos, incrementando 5 veces el riesgo de sufrir un accidente cerebrovascular. En los últimos años, en Latinoamérica se ha alertado sobre los crecientes eventos de accidentes cerebrovasculares debidos a FA ${ }^{[2]}$. Los pacientes con FA son hospitalizados dos veces más que los pacientes sin FA, y su calidad de vida se ve disminuida. Asimismo, la FA genera importantes gastos a los sistemas de salud, solo en Estados Unidos, el gasto anual del tratamiento llega a los US\$26 billones ${ }^{[3]}$. La carga financiera se agudiza en regiones en vía de desarrollo, como Latinoamérica.

La FA se clasifica en paroxística, cuando su duración es menor a 7 días, en persistente, cuando la fibrilación se sostiene por más de 7 días, o en persistente de larga duración cuando la fibrilación continua por más de un año. Para pacientes con FA sintomática el tratamiento se enfoca en restablecer y mantener el ritmo sinusal, en este caso, el manejo se realiza mediante medicamentos antiarrítmicos o a través de la terapia de ablación por radiofrecuencia utilizando catéteres intracavitarios ${ }^{[3,4]}$.

El tratamiento por ablación auricular consiste en la generación de lesiones en el tejido que bloquean la propagación del impulso eléctrico para prevenir la formación y el mantenimiento de la conducción fibrilatoria [5]. La ablación por catéter, a diferencia de la medicación antiarrítmica, se convierte en una opción para curar definitivamente la FA, y puede evitar los efectos secundarios del tratamiento farmacológico a largo plazo.

Estudios clínicos han mostrado que la ablación por radiofrecuencia es efectiva en $70 \%$ de los pacientes con FA paroxística [6]. La guía de la Asociación Americana del Corazón para el manejo de pacientes con fibrilación auricular recomienda la ablación por catéter, como terapia de primera línea, en pacientes con FA paroxística sintomática, que son refractarios o intolerantes al menos a una medicación antiarrítmica [3]. Sin embargo, en pacientes con FA persistente la tasa de éxito, no alcanza el 50\% ${ }^{[7,8]}$. En la misma guía ${ }^{[3]}$, la terapia de primera línea en FA persistente es la medicación antiarrítmica, y solo recomienda considerar la ablación en pacientes con FA persistente cuando es refractario o intolerante al menos a una medicación antiarrítmica.

La baja tasa de éxito de la ablación en FA persistente, puede ser una consecuencia del poco entendimiento de los mecanismos que generan y perpetúan la arritmia ${ }^{[9]}$. La mayoría de los procedimientos de ablación para pacientes con FA están basado en consideraciones anatómicas, donde el procedimiento estándar es el aislamiento de venas pulmonares ${ }^{[10]}$. Por otro lado, existen propuestas para realizar ablación guiada por el análisis de las señales adquiridas con los catéteres intracavitarios, dichas señales se conocen como electrogramas (EGM). Estas estrategias están orientadas a la localización de los substratos arritmogénicos, sin embargo, no han sido universalmente aceptadas. Las principales propuestas que han sido probadas en la práctica clínica son la ablación guiada por electrogramas auriculares fragmentados complejos (CFAE) y la ablación de rotores guiados por mapeo panorámico (FIRM) ${ }^{[11]}$.

Existen estrategias que se basan en la caracterización y clasificación de los electrogramas. Por ejemplo, mediante el cálculo de la frecuencia dominante ${ }^{[12]}$ o el estudio de la entropía ${ }^{[13,14]}$. Sin embargo, ninguno de estos métodos ha encontrado un consenso en la práctica médica, debido en parte a la dificultad de reproducir los resultados con tasas de éxito similares ${ }^{[15]}$.

La problemática a la que se han enfrentado estos métodos para su aceptación es que, a diferencia de la señal ECG, no existe una descripción del todo clara de 
los patrones que se pueden encontrar en las señales EGM y no hay un consenso entre los especialistas que defina claramente los tipos de electrogramas asociados a sitios arritmogénicos, lo cual hace que la ablación en FA siga limitada al aislamiento de las venas pulmonares y que cuando se realiza la ablación guiada por electrogramas, esta dependa de la experiencia del electrofisiólogo ${ }^{[16,17]}$.

Algunos estudios han mostrado que diferentes técnicas para la identificación de electrogramas no identifican necesariamente las mismas áreas de ablación, por lo cual, la caracterización precisa de EGM auriculares que pueden indicar un área particular como objetivo de ablación es actualmente un tema de debate ${ }^{[18]}$.

A partir de esta problemática, el objetivo de este artículo es presentar el estado actual de las estrategias de ablación guiadas por electrogramas y un estado del arte de las propuestas que se reportan en la literatura para desarrollar nuevos métodos que permitan guiar la ablación a partir del procesamiento y análisis de las señales EGM.

El artículo está organizado de la siguiente forma: primero se presentan las tecnologías utilizadas en la práctica clínica para el mapeo electroanatómico, que es la base de los procedimientos de ablación; luego se presentan las principales técnicas de ablación utilizadas en la práctica clínica; posteriormente el artículo se enfoca en presentar las principales propuestas publicadas en la literatura para el análisis de electrogramas, las principales técnicas de procesamiento de señal para caracterización de electrogramas y su aplicación para guiar procedimientos de ablación. Por último, se resumen los esquemas que se han planteado para clasificar los electrogramas de acuerdo con su morfología y se describen las propuestas orientadas a realizar una discriminación de los electrogramas a partir de técnicas de reconocimiento de patrones, incluyendo técnicas de aprendizaje supervisado y semi-supervisado.

\section{MAPEO INTRACARDIACO COMO BASE PARA LOS PROCEDIMIENTOS DE ABLACIÓN}

El mapeo electro-anatómico es un procedimiento que permite identificar, caracterizar y localizar una arritmia y es la base para la realización de las terapias de ablación. Los catéteres cuentan con uno o varios electrodos metálicos expuestos en el extremo. Los EGM son adquiridos a través del contacto directo de los electrodos con el tejido endocárdico ${ }^{[19]}$.

En el mapeo electro-anatómico es necesario combinar la información eléctrica contenida en las señales EGM con información anatómica de las aurículas. Ésta se obtiene a partir de lecturas de posicionamiento de la punta del catéter mediante sistemas de triangulación a partir de campos eléctricos o magnéticos.

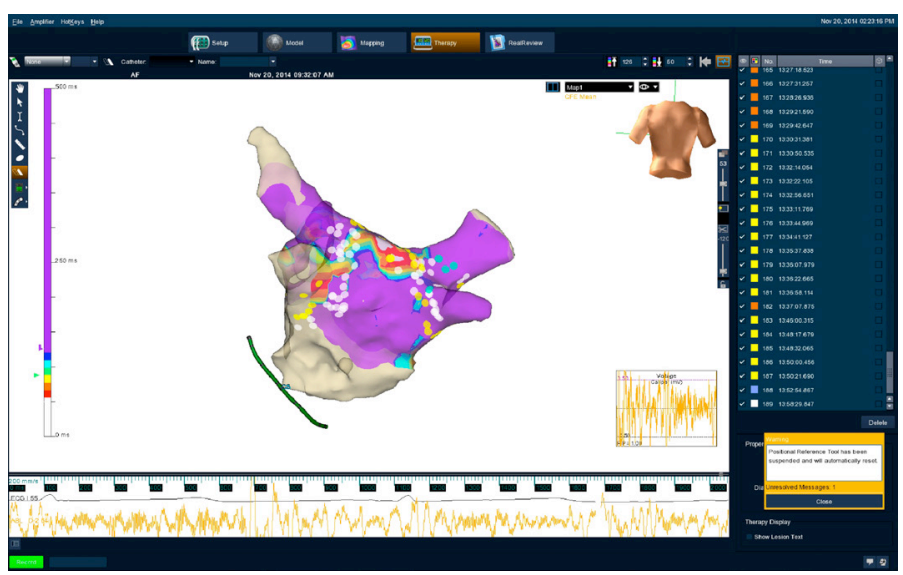

FIGURA 1. Vista posterior de un mapa electroanatómico que representa la reconstrucción 3D de la aurícula izquierda de un paciente. La información eléctrica extraída de los electrogramas se representa en un mapa de color.

Los sistemas de mapeo electroanatómico más utilizados en la práctica clínica a nivel mundial son el EnSite Velocity NavX de St. Jude Medical y el Carto de Biosense Medical, filial de Johnson and Johnson. Estos sistemas proveen la tecnología necesaria para localizar los catéteres en interior de la aurícula y construir mapas anatómicos 3D que permiten al especialista visualizar en qué estructura anatómica auricular está posicionado el catéter ${ }^{[20]}$. Igualmente, los catéteres pueden registrar 
EGM unipolares o bipolares al estar en contacto con la aurícula (La Figura 1 muestra un mapa electroanatómico extraído del sistema NavX).

\section{TÉCNICAS DE ABLACIÓN GUIADAS ANATOMICAMENTE Y SUS LIMITACIONES}

El procedimiento estándar de ablación en FA consiste en el aislamiento eléctrico de las venas pulmonares (PVI). Se realiza aplicando ablación circunferencial o ablación segmentada, con el objetivo de desconectar eléctricamente las venas pulmonares del sistema de propagación del potencial de acción del miocardio. Para su realización, se utilizan los sistemas de mapeo 3D, para guiar los catéteres al interior de la estructura de las aurículas sin necesidad de buscar substratos arritmogénicos mediante el análisis de EGM.

El procedimiento de PVI se basa en la evidencia que la mayoría de los focos ectópicos que desencadenan episodios de FA se localizan en las venas pulmonares ${ }^{[21]}$. Sin embargo, la FA puede ser iniciada y sostenida por otros gatillos y mecanismos, especialmente en casos de FA persistente. Estudios recientes han demostrado la presencia de circuitos fibrilatorios que generan una conducción de alta frecuencia alrededor de un vértice ${ }^{[22,23]}$. Basados en esto, Jalife et al. propusieron la hipótesis del rotor, según la cual, la FA es sostenida por circuitos de micro-reentrada que giran alrededor de un punto de singularidad ${ }^{[22]}$. Los rotores pueden ser iniciados por latidos ectópicos provenientes de las venas pulmonares, y posteriormente se auto sostienen.

A diferencia de la hipótesis de ondas circulares sugerida a principios del siglo XX, la hipótesis del rotor asegura que no se requiere la presencia de un obstáculo anatómico para que se genere un rotor. Se han estudiado rotores mediante el registro de la propagación del potencial de acción a partir de métodos ópticos en cultivos celulares ${ }^{[24]} \mathrm{y}$ en corazones aislados de animales [23]. Recientemente, Narayan et al. han logrado observar rotores utilizando un catéter tipo balón con 64 electro- dos, generando una mayor evidencia sobre la existencia de rotores en humanos con FA ${ }^{[25]}$.

Por otra parte, Allessie et al., introducen la hipótesis del mecanismo de la "doble capa", la cual indica que la FA persistente se sostiene debido a una disociación progresiva endo-epicárdica, que va convirtiendo el miocardio en una doble capa de conducción que se autoalimenta constantemente ${ }^{[26]}$. Esta hipótesis es sustentada con base a estudios electrofisiológicos en pacientes con FA persistente, que indican que las ondas fibrilatorias observadas son de origen "focal" pero sin la presencia de un área con actividad focal sostenida ${ }^{[27]}$, es decir, existe un avance disruptivo de la onda de propagación entre el epicardio y el endocardio. Aunque esta hipótesis explicaría por qué la ablación de FA persistente no es exitosa ${ }^{[28]}$, requeriría de un mapeo endocárdico y epicárdico simultáneo para observarse [29], lo cual es inviable en humanos con la tecnología disponible actualmente.

Otras variantes de la ablación guiada por estructuras anatómicas, sugiere la generación de líneas de ablación adicionales en la aurícula izquierda. Los sitios más comunes para la generación de estas líneas son la pared superior de la aurícula izquierda conectando las lesiones de las venas pulmonares superior derecha e izquierda y la región de tejido entre la válvula mitral y la vena pulmonar inferior izquierda ${ }^{[30]}$. De esta forma se busca eliminar sitios críticos en el mantenimiento de la FA diferentes a las venas pulmonares.

Un procedimiento de ablación óptimo debería generar la cantidad mínima de líneas de ablación necesarias para eliminar los substratos arritmogénicos que generan o sostienen la arritmia. Sin embargo, en las estrategias guiadas anatómicamente, se generan líneas de ablación que no necesariamente garantizan la eliminación de estos puntos críticos y que, por el contrario, pueden lesionar parte del tejido sano. Para evitar este problema, se han propuesto métodos para realizar 
la ablación guiada por EGM en los cuales el resultado del análisis de los EGM es utilizado para detectar sitios objetivos de ablación.

\section{TÉCNICAS DE ABLACIÓN GUIADAS POR ELECTROGRAMAS}

A pesar del entendimiento parcial de los mecanismos de generación y perpetuación de la FA, se han propuesto múltiples métodos para realizar la ablación guiada por electrogramas (EGM), entre ellos el mapeo de electrogramas fragmentados y el mapeo panorámico. Es necesario tener en cuenta que debido a la naturaleza desorganizada de la propagación del potencial de acción en FA, es muy difícil hacer el seguimiento de dicha propagación por medio de mapas de activación como se hace en casos de taquicardia y flutter auricular ${ }^{[3]}$.

\section{Electrogramas complejos fragmentados}

Una de las estrategias para guiar los procedimientos de ablación basados en los EGM, es el análisis de los llamados electrogramas auriculares fragmentados complejos (CFAE) ${ }^{[31]}$. La ablación de CFAE es uno de los métodos más utilizados en la práctica clínica, gracias a que ha estado disponible en los equipos comerciales de mapeo cardíaco más usados (EnSite Velocity NavX de St. Jude Medical y Carto de Biosense Medical). Esta aproximación diagnóstica inició en 2004 con Nademanee et al. ${ }^{[31]}$ quien definió genéricamente los CFAE como: 1) Potenciales de EGM auriculares fragmentados que poseen 2 o más deflexiones y/o perturbaciones de la línea base con deflexiones continuas y activación prolongada. 2) EGM auriculares con una longitud de ciclo muy corta ( $\leq 120 \mathrm{~ms}$ ) promediado sobre 10 segundos de registro. La Figura 2 ilustra un ejemplo de señal considerada normal y de una señal CFAE.

Nademanee et al. ${ }^{[31]}$ proponen identificar y tratar las áreas con CFAE como sitios susceptibles de ablación. Sin embargo, estudios posteriores han reportado resultados contradictorios ${ }^{[32,33]}$ y por ello este método tiene

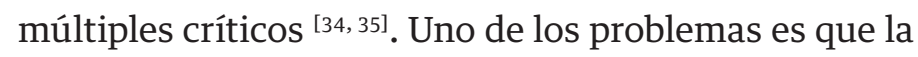
definición de CFAE es muy general y no distingue entre diferentes morfologías presentes en los electrogramas fragmentados, por lo tanto, se caracteriza como CFAE a una familia muy amplia de señales que no necesariamente están relacionadas con substratos arritmogénicos ${ }^{[36]}$. Esto conlleva a una interpretación subjetiva de cuáles son los EGM fragmentados que verdaderamente pueden garantizar que el procedimiento de ablación sea exitoso ${ }^{[16,37]}$.

Los estudios realizados para comparar las estrategias propuestas de ablación en FA persistente presentan mucha variación en los resultados y por lo tanto actualmente no es claro qué tipo de ablación presenta mejor desempeño. Por ejemplo, mientras los estudios de Nademanee et al. reportan un éxito del 91\% sin recurrencia durante un año ${ }^{[31]}$, Oral et al. no lograron reproducir dichos resultados y reportan una tasa de éxito solo del $40 \%{ }^{\text {[32] }}$, mientras que Elayi et al. alcanzaron un $69 \%$ de éxito ${ }^{[38]}$. Los estudios que utilizan PVI con ablación lineal reportan tasas de éxito que oscilan entre 11\% y 74\%. Así mismo, estudios que describen una estrategia de ablación secuencial (PVI + líneas + CFAE), reportan tasas de éxito entre $38 \%$ y $62 \%{ }^{[7,39]}$. En general, la tasa de éxito promedio en FA persistente utilizando PVI + CFAE es de $47 \%$, lo cual es muy cercano a los reportes para ablación donde solo se hace PVI (40\%) ${ }^{[40]}$. Recientemente, Verma et al. en el estudio STAR-AF II, el cual incluyó el seguimiento de 549 pacientes, concluyeron que bajo las técnicas actuales lo más recomendable es realizar únicamente PVI ${ }^{[35]}$.

Respecto a lo anterior, es necesario enfatizar que los descriptores utilizados para detectar CFAE, implementados en los dispositivos de mapeo intracardiaco utilizados en la mayoría de los estudios, están basados en la definición de Nademanee, que como se mencionó, es una definición cualitativa que no diferencia los patrones presentes en las señales EGM fragmentadas. Por otra parte, la poca reproducibilidad de los resulta- 
dos se debe principalmente a que la definición de CFAE es muy amplia, y hace necesario que el electrofisiólogo evalúe visualmente las señales y a partir de su experiencia, seleccione los CFAE que pueden indicar un sitio objetivo de ablación ${ }^{[41,42]}$. Lo anterior implica que la clasificación binaria de señales EGM entre CFAE y no-CFAE es insuficiente. Este hecho, influye de una manera importante en los diferentes estudios clínicos.
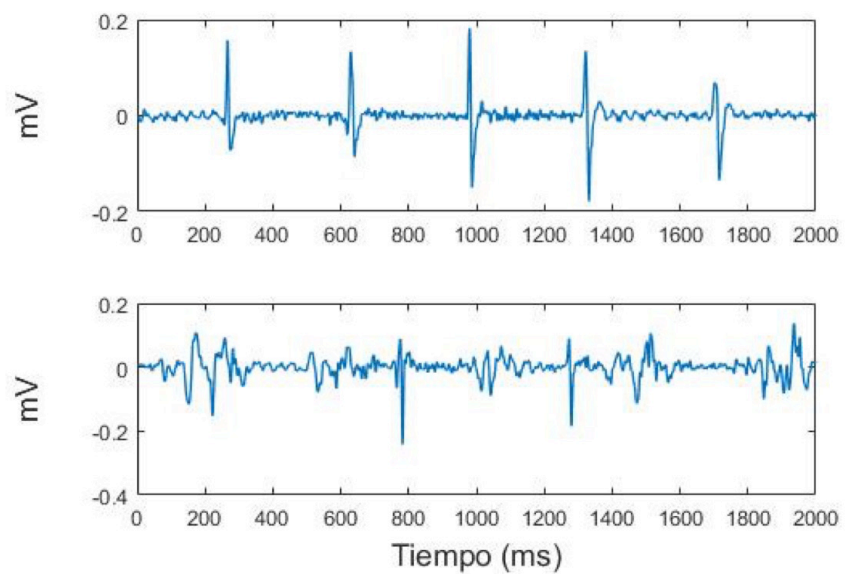

FIGURA 2. Ejemplo de señales EGM. Arriba una señal donde se observan las activaciones locales de forma organizada y abajo una señal fragmentada (CFAE).

Si bien la ablación guiada por CFAE tuvo un auge importante en los últimos años, su utilización ha sido cuestionada recientemente por los estudios STARTAF-II y CHASE AF ${ }^{[35,43]}$. En respuesta a esto, Narayan et al. han propuesto un nuevo método de ablación guiado por mapeo panorámico, con el cual se realiza un seguimiento a la propagación de la conducción eléctrica a través de la aurícula ${ }^{[44]}$. El método propuesto y desarrollado por Narayan et al. se basa en un mapeo panorámico intracardiaco de las 2 aurículas, utilizando catéteres tipo balón con 64 electrodos. Dicho catéter cuenta con 8 líneas longitudinales, cada una con 8 electrodos, con lo cual se cubre casi toda la aurícula. Las señales son adquiridas y procesadas, mediante un patentado basado en mapas de fase, para determinar los patrones de propagación eléctrica presentes en cada paciente. Utilizando mapas isocrónicos, se determina el centro del rotor como zona objetivo de ablación para revertir la arritmia ${ }^{[25]}$. El método recibe el nombre de Focal Impulse and Rotor Modulation (FIRM) ${ }^{\text {[11] }}$. A partir de dicho método, un estudio prospectivo realizado en 92 pacientes (llamado CONFIRM) reportó la detección de rotores en humanos como fuentes conductoras de la arritmia ${ }^{[45]}$.

Este esquema fue desarrollado bajo la hipótesis de la existencia de rotores estables, lo cual ha sido debatido y permanece como tema abierto ${ }^{[26]}$. Adicionalmente, a pesar de los resultados publicados por Narayan et al., varios autores cuestionan el método FIRM. Allesie y de Groot, sostienen que los resultados de Narayan et al. no son concluyentes y que todavía se deben realizar estudios adicionales en otros centros para validarlos ${ }^{[29]}$. Sostienen además que el método de Narayan utiliza un mapeo de baja resolución (64 electrodos), respecto a otros estudios en los que se ha realizado mapeo epicárdico en humanos con 128 y 256 electrodos, en los cuales no se observaron rotores estables en humanos ${ }^{[26,46]}$. Buch et al. ${ }^{[47]}$ realizaron un estudio de cohortes en dos centros médicos de Estados Unidos con 43 pacientes en el cual utilizaron el método FIRM encontrando una eficacia muy pobre, en contravía de lo reportado por los estudios de Narayan et al. Ellos concluyen que aún se requieren una serie de estudios aleatorizados para comprobar la eficacia y utilidad critica del método FIRM.

\section{PROPUESTAS DE MAPEO BASADO EN LA CARACTERIZACIÓN DE LOS ELECTROGRAMAS}

En FA persistente la morfología de los electrogramas es muy desorganizada y dificulta la detección de las ondas de activación, lo que conlleva a imprecisiones en la localización de los substratos arritmogénicos a partir del mapeo panorámico. Por otra parte, las estrategias basadas en CFAE, dependen en gran medida de la capacidad del especialista de discriminar que tipo 
CFAE deben ser seleccionados para guiar la ablación. Como alternativa, en la literatura se han generado varias propuestas basadas en el procesamiento de las señales EGM con herramientas matemáticas que buscan caracterizar las señales por medio de índices o descriptores que no dependen del concepto de CFAE definido por Nademanee, y que puedan cuantificar las formas de onda encontradas en estas señales. Características basadas en la morfología de la señal ${ }^{[48]}$, en el análisis en el dominio de la frecuencia ${ }^{[49]}$, o en descriptores no lineales ${ }^{[50]}$, han mostrado resultados prometedores en estudios sobre modelos computacionales o sobre modelos animales, pero todavía requieren ser validados antes de su aplicación en la práctica clínica. A continuación, se describen los desarrollos más relevantes en este sentido.

Estudios clínicos han mostrado que los sitios anatómicos que representan substratos de FA están caracterizados por señales EGM con un alto grado de desorganización y ondas de activación de alta frecuencia [51]. De acuerdo a esto, algunas propuestas para el procesamiento de la señal EGM están orientadas a calcular índices que cuantifican la frecuencia de las ondas de activación y la longitud del ciclo, tanto de los potenciales regulares como de los potenciales fragmentados. Posteriormente los algoritmos se han orientado a la caracterización del nivel de organización de la señal.

Para medir el grado de organización espacial de la ondas de activación durante FA, Botteron y Smith ${ }^{[52]}$ propusieron un método de pre-procesamiento que genera una forma de onda variante en el tiempo proporcional a la amplitud de los componentes de alta frecuencia en el EGM auricular; esta señal resultante corresponde a la envolvente del EGM. Este método ha sido utilizado para calcular diferentes índices, basados en la aplicación de la transformada de Fourier, para encontrar el espectro de potencia de la envolvente de la señal EGM: frecuencia dominante (DF), índice de organización (OI) e índice de regularidad (RI) ${ }^{[53,54]}$.
Sin embargo, existen autores que han discutido la utilidad práctica del análisis del DF, ya que es sensible al método de adquisición de la señal, a la relación señal a ruido (SNR), a la despolarización de campo lejano ventricular y a la complejidad de la señal ${ }^{[55,56]}$. Faes et al.

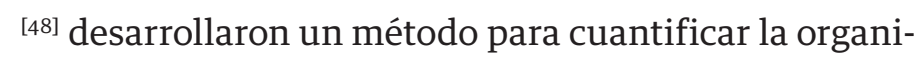
zación de EGM bipolares simples, adquiridos en una aurícula humana durante FA. El algoritmo se basa en la comparación entre pares de ondas de activación para estimar su similitud morfológica, y retorna una medida llamada índice de similitud, el cual mide el grado de repetitividad en el tiempo de las ondas de activación.

Algunos autores han propuesto métodos basados en transformadas tiempo-frecuencia para la caracterización de la señal EGM o para la segmentación de la señal con el fin de detectar las ondas de activación y los segmentos con actividad eléctrica, ya sea de campo cercano -activaciones que representan la activación localo de campo lejano -que representan las activaciones de zonas diferentes a las inmediaciones del catéter [57]. Houben, de Groot y Allessie ${ }^{[57]}$ utilizaron la descomposición wavelet en EGM unipolares en FA para la detección automática de ondas de activación locales durante FA, a partir de las cuales se cuantifican los potenciales primarios y el grado de fragmentación de los EGM. Kremen et al. ${ }^{[58]}$ propusieron un método basado en la transformada wavelet discreta para la detección de los segmentos con actividad eléctrica. De acuerdo a esta propuesta, se identificó que en señales EGM con una frecuencia de muestreo de $1000 \mathrm{~Hz}$, el coeficiente de detalle 3 contiene información relevante para la detección de las ondas de activación.

Por otro lado, algunos estudios han propuesto la utilización de métodos no lineales para el estudio del fenómeno de fragmentación en los EGM auriculares [59, 60]. Ganesan et al. ${ }^{[61]}$ evaluaron la aplicación de la entropía de Shannon para detectar electrogramas fragmentados en un modelo computacional 2D, en modelos in-vivo (ratas y ovejas) y en pacientes con FA persistente. En 
este estudio encontraron que las áreas de alta entropía están relacionadas con rotores en los modelos computacionales y los modelos in-vivo, pero los resultados en humanos mostraron áreas de alta entropía que no están relacionadas con los mapas de CFAE generados por los equipos comerciales, y no fue posible distinguir a qué tipo de substrato se podrían asociar.

Han sido propuestas medidas de entropía basadas en la teoría de dinámica no lineal como la entropía aproximada (ApEn) y la entropía Sample (SampEn) para la evaluación del nivel de fragmentación de señales EGM, y se ha evaluado su relación con la detección de rotores en modelos computacionales que simulan la conducción fibrilatoria auricular ${ }^{[62,}{ }^{63]}$. También se ha propuesto la utilización de métodos de análisis multifractal en la evaluación de diferentes niveles de fragmenta-

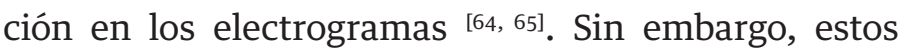
métodos requieren nuevos estudios para su validación y aún no están siendo utilizados en la práctica clínica.

\section{ESQUEMAS DE CLASIFICACIÓN}

\section{PARA EL ANÁLISIS DE ELECTROGRAMAS}

Los patrones de propagación en FA tienen una alta complejidad por lo que caracterizar las señales que se adquieren a través de un solo descriptor, por ejemplo, la frecuencia dominante, la entropía de Shannon o la entropía aproximada, puede no ser suficiente. Teniendo en cuenta esto, algunas investigaciones han abordado el tema como un problema de reconocimiento de patrones. Se han propuesto esquemas basados en modelos de aprendizaje supervisado para detectar los electrogramas con alto nivel de fragmen-

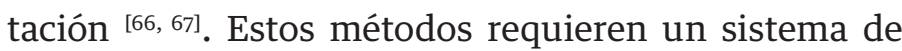
clasificación abalado por expertos que permita generar un conjunto de señales etiquetadas para ser utilizadas en el proceso de entrenamiento de los algoritmos de aprendizaje supervisado. Aunque no existe un consenso en cuanto a la clasificación de los electrogramas, en la literatura se han planteado algunos esquemas de clasificación, basados principalmente en el nivel de organización de la señal y en el porcentaje de actividad eléctrica presente en la señal. A continuación, se resumen estos esquemas, los cuales sirven como base para los métodos de reconocimiento de patrones.

\section{Clasificación de acuerdo al esquema de Wells}

Uno de los primeros sistemas de clasificación de señales EGM fue propuesto por Wells et al. [68] quienes distinguieron los EGM auriculares en cuatro tipos basados en la presencia de activaciones en el EGM y la naturaleza de su línea base:

, Tipo I: Ondas de activación discretas separadas por una línea base isoeléctrica libre de perturbación.

, Tipo II: Ondas de activación con varias perturbaciones y línea base no isoeléctrica.

, Tipo III: EGM en los cuales se dificulta la identificación de ondas de activación o la distinción de una línea base definida.

1 Tipo IV: Definido como el tipo III en el que adicionalmente se presentan periodos alternantes con características del tipo I o II.

Uno de los principales problemas de esta clasificación es que no tiene un criterio definido y cuantificado para evaluar que se puede considerar una perturbación de la línea base, y no tiene en cuenta diferentes morfologías que puedan aparecer en las ondas de activación, de tal forma que la clasificación se basa en parámetros subjetivos. Por otro lado, los EGM tipo IV están definidos como una señal que cambian su morfología por tramos. Esto dificulta su análisis de tal forma que usualmente solo se consideran los tres primeros tipos.

\section{Clasificación escalada en cuatro niveles de fragmentación}

Schilling et al. y Kremen et al. ${ }^{[69,70]}$ han introducido un sistema de clasificación que puede ser considerado una modificación del criterio de Wells, basado en 4 grupos, 
de tal forma que los grupos con más alto nivel, presentan mayor nivel de desorganización o fragmentación:

, Co: EGM auriculares no fragmentados con alta frecuencia.

, C1: EGM fragmentados con actividad periódica (nivel bajo de fragmentación).

, C2: Mezcla de actividad periódica fragmentada y EGM no fragmentados con actividad no periódica (nivel intermedio de fragmentación).

, C3: EGM auriculares de alta frecuencia y con actividad continúa (no se pueden diferenciar ondas de activación discretas de forma regular, lo cual se considera un nivel alto de fragmentación).

Este esquema de clasificación contempla la presencia de diferentes tipos de morfologías en la señal EGM, sin embargo, los criterios no son cuantitativos y esta clasificación depende en gran medida de criterios subjetivos del electrofisiólogo que evalúe las señales.

\section{Clasificación según la duración de la actividad fragmentada}

Hunter et al. ${ }^{[71]}$ propusieron una clasificación de las señales en 5 tipos, fundamentada, principalmente, en la duración de la actividad fragmentada, con lo que se incluye un criterio adicional cuantitativo para definir los grupos:

, Grado 1: Presencia de actividad fragmentada ininterrumpida en más del 70\% de la señal, que se caracteriza por la presencia de deflexiones continúas sin pausa en la línea isoeléctrica, con episodios perdurables mayores a 1 segundo. Se consideran continuas si la separación entre deflexiones es menor a $70 \mathrm{~ms}$.

, Grado 2: Actividad fragmentada interrumpida, cuando la actividad fragmentada ocupa más del $70 \%$ de la señal, pero no hay episodios perdurables.
, Grado 3: Actividad fragmentada intermitente, cuando la actividad fragmentada ocupa entre 30 y $70 \%$ de la señal.

, Grado 4: EGM complejos, definidos como potenciales discretos ( $\leq 70 \mathrm{~ms}$ ) y complejos ( $\geq 5$ cambios de dirección), con cualquier tipo de actividad fragmentada ocupando menos del 30\% de la señal (de otro modo, estaría considerada como de grado 3 ).

, Grado 5: Electrogramas normales, potenciales discretos (separación $\geq 70$ ms) y simples ( $<5$ cambios de dirección).

Esta clasificación, aunque incluye criterios cuantitativos, no ha logrado convertirse en un estándar clínico. Algunas limitaciones de este esquema es que no evalúa la morfología de las ondas de activación y no se ha definido una relación directa de cada grupo con los sustratos arritmogénicos críticos en la perpetuación de las arritmias.

Se pueden encontrar algunos paralelismos entre los diferentes tipos de clasificación, ya que estos sistemas se basan en la valoración del nivel de desorganización de la señal, en la Tabla 1 se presenta una descripción de 5 clases de EGM que resume las asociaciones entre las clases definidas en los sistemas descritos anteriormente. La Figura 3, ilustra un ejemplo de señal EGM para cada una de estas 5 clases.

\section{MÉTODOS DE APRENDIZAJE SUPERVISADO PARA LA CLASIFICACIÓN DE LOS ELECTROGRAMAS}

Con el fin de utilizar modelos que combinen información de varias características simultáneamente, se han propuesto nuevos métodos basados en esquemas de reconocimiento de patrones. Algunos estudios han propuesto sistemas de reconocimiento de patrones para la detección de diferentes niveles de fragmentación basado en alguno de los esquemas descritos en la sección anterior. 
El problema de la detección de patrones en EGM, ha sido abordado principalmente mediante el uso de algoritmos de clasificación basados en aprendizaje supervisado. Nollo et al. ${ }^{[72]}$ presentaron un sistema automático para el análisis y clasificación de FA utilizando una máquina de soporte vectorial (SVM) para clasificar entre EGM tipo I, II y III de acuerdo al criterio de Wells. Ellos trabajaron con una base de datos limitada a 100 señales y obtuvieron una exactitud de $97.7 \%$.

Kremen et al. ${ }^{[66]}$ propuso un algoritmo para la detección automática de electrogramas fragmentados utilizando un método basado en la teoría de grupos de modelos de evolución adaptativa llamado GAME (Group of Adaptive Models Evolution), la cual se basa en redes neuronales artificiales. Para la caracterización de la señal fue utilizada la transformada wavelet además de otros algoritmos para la extracción de características basados en el dominio del tiempo, el dominio de la frecuencia y en dinámica no lineal. Utilizaron procedimientos de optimización para encontrar la mejor configuración posible de los algoritmos y las características para maximizar la efectividad de la etapa de clasificación. Los algoritmos desarrollados por Kremen et al. fueron validados en una base de datos etiquetada por expertos de acuerdo al criterio de Schilling et al. Los resultados mostraron un promedio de error en la clasificación para todas las clases del 6\%, y un error de clasificación de alto grado de fragmentación de $9 \%$.

TABLA 1. Evaluación de la relevancia de las características utilizando el índice FDR. En negrilla se presentan las características seleccionadas para la implementación del clasificador.

\begin{tabular}{|c|c|c|c|c|}
\hline Nueva clase & Descripción/clasificación & Hunter & Schilling-Kremen & Wells \\
\hline $\mathrm{A}$ & $\begin{array}{l}\text { EGM con activaciones discretas, } \\
\text { periódicas y simples. }\end{array}$ & Grupo 5 & Clase 0 & \multirow{2}{*}{ Tipo 1} \\
\hline $\mathrm{B}$ & $\begin{array}{l}\text { EGM con activaciones discretas, } \\
\text { periódicas, pero fragmentadas. }\end{array}$ & Grupo 4 & Clase 1 & \\
\hline $\mathrm{C}$ & $\begin{array}{l}\text { EGM con activaciones discretas } \\
\text { pero desorganizadas con actividad } \\
\text { no periódica. }\end{array}$ & Grupo 3 & Clase 2 & Tipo 2 \\
\hline $\mathrm{D}$ & $\begin{array}{l}\text { EGM con actividad desorganizada, } \\
\text { con segmentos de activación } \\
\text { continua y segmentos con actividad } \\
\text { discreta pero no periódica y } \\
\text { fragmentada. }\end{array}$ & Grupo 2 & \multirow[t]{2}{*}{ Clase 3} & \multirow[t]{2}{*}{ Tipo 3} \\
\hline $\mathrm{E}$ & $\begin{array}{l}\text { EGM auriculares de alta frecuencia } \\
\text { y con actividad continúa. }\end{array}$ & Grupo 1 & & \\
\hline
\end{tabular}

Schilling et al. ${ }^{[67]}$ implementaron un clasificador basado en un árbol de decisión difuso. Este sistema fue entrenado con una base de datos de 429 señales etiquetadas en 4 clases. En total utilizaron 18 características entre las que se incluyen descriptores en el dominio del tiempo, información a partir del espacio de fase, descriptores basados en wavelet, características basadas en la amplitud y el índice de similitud propuesto por Faes et al.
El trabajo reporta una tasa de aciertos para clasificar entre 4 clases del $80.6 \%$. Duque et al. ${ }^{[73]}$ reportan un esquema basado en un clasificador K-NN y una combinación de 41 características seleccionadas por medio de un algoritmo genético. Las características incluyen descriptores en el tiempo, la frecuencia, tiempo-frecuencia y características no lineales. Este clasificador obtuvo una tasa de aciertos de $91 \%$ trabajando en la misma base de datos de Schilling et al. 

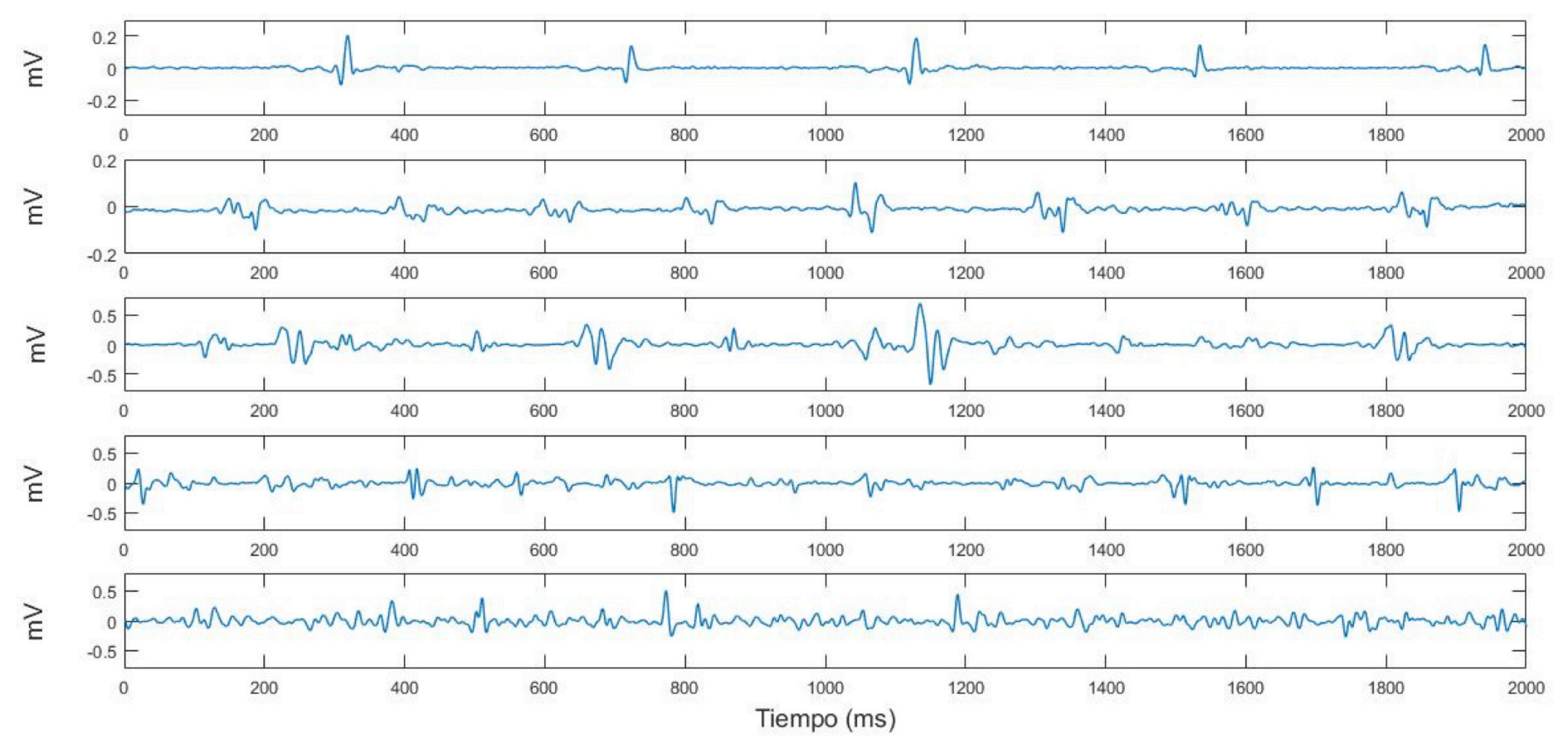

FIGURA 3. Patrones de señales EGM de acuerdo a las clases definidas en la Tabla 1.

La metodología basada en clasificadores supervisados podría ser incorporada en un sistema de mapeo para facilitar la identificación de potenciales fragmentados y así guiar los procedimientos de ablación, sin embargo, ninguno de los esquemas descritos anteriormente ha sido aplicado en la práctica clínica. Una de las limitaciones radica en que estos sistemas dependen de un proceso de entrenamiento a partir de señales etiquetadas manualmente bajo los parámetros definidos por un determinado grupo de investigación, lo cual, debido al poco conocimiento de las morfologías presentes en las señales EGM y la falta de un estándar para clasificar las señales, hace que la solución encontrada esté sesgada por las propiedades estadísticas de la base de datos utilizada en el entrenamiento, y por la subjetividad del grupo de investigación que etiqueta las señales.

Otra limitación, es que hasta hoy no se ha logrado hacer una relación precisa entre las diferentes clases de electrogramas con los substratos arritmogénicos, y, por lo tanto, no ha sido posible validar en la clínica los procedimientos de ablación guiados por la clasifica- ción de electrogramas. Una de las principales limitaciones para el desarrollo de técnicas que guíen la ablación es que las tecnologías actuales no permiten hacer un seguimiento preciso de la propagación del potencial de acción sobre la aurícula humana, dificultando la identificación directa del patrón de propagación real en cada paciente.

\section{MÉTODO DE APRENDIZAJE SEMI-SUPERVISADO PARA GUIAR PROCEDIMIENTOS DE ABLACIÓN}

Con el fin de superar las limitaciones presentes en los métodos de reconocimiento de patrones basados en aprendizaje supervisado, Orozco-Duque et al. han propuesto un método de mapeo electro-anatómico basado en técnicas de agrupamiento semi-supervisado que permite la localización sobre la superficie auricular de las diferentes morfologías presentes en las señales EGM ${ }^{[74]}$. Este método, llamado FEPMAP (fractionated-electrogram-patterns-mapping), puede ser aplicado bajo las condiciones en que se realiza el mapeo electroanatómico, utilizando equipos comerciales como los equipos NavX y Carto. 
La ventaja del esquema semi-supervisado, respecto a los esquemas supervisados, es que tiene una menor dependencia a las propiedades estadísticas del conjunto de señales etiquetadas utilizadas como información externa. El método FEPMAP, permite realizar una evaluación paciente-específico, para lo cual sólo se utiliza una pequeña cantidad de señales etiquetadas correspondientes a la clase 0 , es decir, señales no fragmentadas. Esto permite una mayor generalización del modelo, por lo que es posible la identificación de morfologías de las ondas de activación con características estadísticas diferentes a las descritas por las bases de datos etiquetadas de las que se dispone. Esta propiedad es importante en la discriminación de las morfologías de los EGM, ya que no existe una definición estándar de fragmentación que describa claramente las clases que pueden ser encontradas en estas señales.

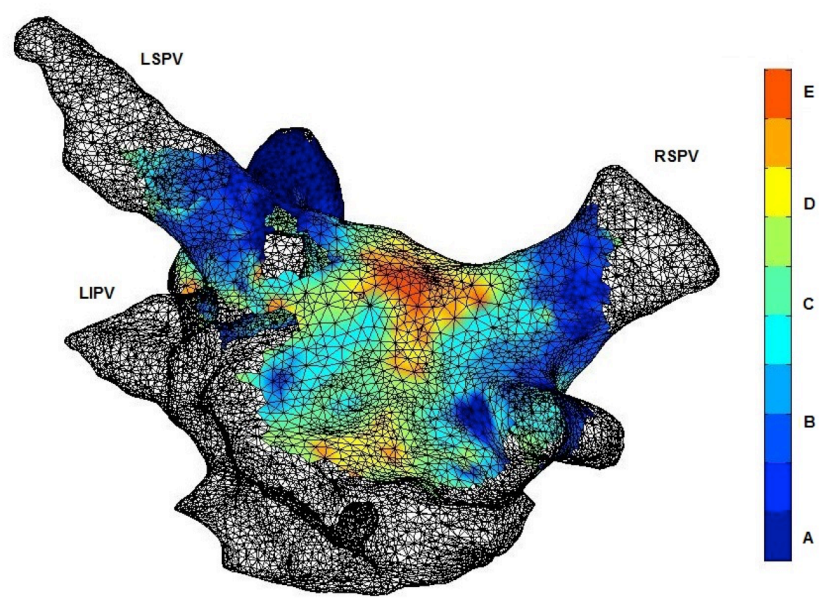

FIGURA 4. Mapa electroanatómico generado con el método FEPMAP. Vista de la pared posterior de la aurícula izquierda de un paciente con FA. La barra de color indica el nivel de desorganización de la señal de acuerdo a las clases definidas en la Tabla 1. La zona roja indica actividad continua y desorganizada y podría estar asociada a un sitio crítico de actividad fibrilatoria.

El método semi-supervisado, permite que la implementación del sistema pueda llevarse a cabo con señales adquiridas en condiciones diferentes a las utilizadas en la base de datos de entrenamiento, por ejemplo, cuando se utilizan diferentes tipos de catéteres. La Figura 4 presenta un mapa generado bajo el método FEPMAP a partir de las señales adquiridas en un paciente de FA persistente con un electrodo circular tipo $\mathrm{LASSO}^{\circledR}$ y utilizando el sistema $\mathrm{NavX}^{\circledR}$ (St Jude Medical, Inc., St. Paul, MN, USA).

\section{CONCLUSIONES}

La ablación en FA persistente continúa siendo un tema de investigación abierto que requiere de un mayor conocimiento en los mecanismos que perpetúan la arritmia y que exige múltiples esfuerzos multidisciplinarios para aumentar las tasas de éxito.

Se han logrado avances significativos en el entendimiento de los mecanismos de la FA en la última década, los cuales han permitido el desarrollo de técnicas de ablación particularmente en los pacientes con FA paroxística. Múltiples estudios clínicos recientes en pacientes con FA persistente indican que agregar el procedimiento de ablación guiada por electrogramas fraccionados o la generación de líneas guiadas anatómicamente no incrementan la tasa de éxito, comparado con realizar exclusivamente el aislamiento de las venas pulmonares.

Estos hallazgos han dado pie a propuestas de modalidades novedosas de mapeo en FA persistente, basadas en métodos y herramientas avanzadas de procesamiento de señales. Los métodos de reconocimiento de patrones tanto supervisados como semi-supervisados podrían usarse como un complemento en la evaluación de sitios objetivos de ablación, ya que permite observar la distribución de las diferentes morfologías de las señales EGM sobre la reconstrucción 3D de la aurícula realizada durante los procedimientos de mapeo.

Estos métodos, aunque ha mostrado evidencia prometedora en el laboratorio respecto a su desempeño en el análisis de señales EGM, deben ser evaluados en estudios clínicos de cohortes amplias para establecer 
la relación entre los patrones encontrados en las señales EGM con los substratos arritmogénicos y los diferentes mecanismos que sostienen la arritmia en el paciente. Igualmente, deben someterse a estudios clínicos para demostrar su eficacia para guiar procedimientos de ablación en pacientes con FA persistente.

\section{AGRADECIMIENTOS}

Al Departamento de Ciencias, Tecnología e Innovación COLCIENCIAS, Colombia; por el soporte en el recurso humano comprometido en plaza de becario de formación doctoral, y el proyecto de investigación contrato 121056933647. 


\section{REFERENCIAS}

[1] Camm AJ, Kirchhof P, Lip GYH, Schotten U, Savelieva I, Ernst S, et al. Guidelines for the management of atrial fibrillation: the Task Force for the Management of Atrial Fibrillation of the European Society of Cardiology (ESC). Eur Heart J [Internet] 2010 [cited 2014 Jan 21];31(19):2369-429. Available from: http://www.ncbi.nlm.nih. gov/pubmed/20802247

[2] Cantú C, True Hills M, Massaro A, Goto S, Hu H-H, Quek DK, et al. Atrial Fibrillation-Related Stroke across Latin America : A Preventable Problem Working Group Recommendations Atrial Fibrillation-Related Stroke across Latin America : A Preventable Problem Working Group Recommendations. 2012.

[3] January CT, Wann LS, Alpert JS, Calkins H, Cigarroa JE, Cleveland JC, et al. 2014 AHA/ACC/HRS Guideline for the Management of Patients With Atrial Fibrillation. J Am Coll Cardiol [Internet] 2014;64(21):e1-76. Available from: https://www.sciencedirect.com/ science/article/pii/S0735109714017409?via\%3Dihub

[4] Calkins H, Kuck KH, Cappato R, Brugada J, Camm a J, Chen S-A, et al. 2012 HRS/EHRA/ECAS expert consensus statement on catheter and surgical ablation of atrial fibrillation: recommendations for patient selection, procedural techniques, patient management and follow-up, definitions, endpoints, and research trial design: a re. Heart Rhythm [Internet] 2012 [cited 2014 Jan 22];9(4):632-696. e21. Available from: http://www.ncbi.nlm.nih.gov/pubmed/22386883

[5] Roten L, Derval N, Jaïs P. Catheter ablation for persistent atrial fibrillation: elimination of triggers is not sufficient. Circ Arrhythm Electrophysiol [Internet] 2012 [cited 2014 Nov 3];5(6):1224-32; discussion 1232. Available from: https://goo.gl/mG1Fhm

[6] Cappato R, Calkins H, Chen S-A, Davies W, Iesaka Y, Kalman J, et al. Updated worldwide survey on the methods, efficacy, and safety of catheter ablation for human atrial fibrillation. Circ Arrhythmia Electrophysiol 2010;3(1):32-8.

[7] Brooks AG, Stiles MK, Laborderie J, Lau DH, Kuklik P, Shipp NJ, et al. Outcomes of long-standing persistent atrial fibrillation ablation: A systematic review. Hear Rhythm 2010;7(6):835-46.

[8] Arbelo E, Brugada J, Hindricks G, Maggioni AP, Tavazzi L, Vardas P, et al. The Atrial Fibrillation Ablation Pilot Study: An European Survey on Methodology and results of catheter ablation for atrial fibrillation conducted by the European Heart Rhythm Association. Eur Heart J 2014;35(22):1466-78.

[9] Mathew ST, Patel J, Joseph S. Atrial fibrillation: mechanistic insights and treatment options. Eur J Intern Med [Internet] 2009 [cited 2014 Nov 3];20(7):672-81. Available from: http://www.ncbi. nlm.nih.gov/pubmed/19818285

[10] Yaksh A, Kik C, Knops P, Roos-Hesselink JW, Bogers a JJC, Zijlstra F, et al. Atrial fibrillation: to map or not to map? Netherlands Hear J [Internet] 2014;22(6):259-66. Available from: http://www.pubmedcentral.nih.gov/articlerender.fcgi?artid $=4031356 \&$ tool $=$ pmcentrez\&rendertype $=$ abstract

[11] Narayan SM, Krummen DE, Enyeart MW, Rappel WJ. Computational Mapping Identifies Localized Mechanisms for Ablation of Atrial Fibrillation. PLoS One 2012;7(9).

[12] Chang S-L, Chen Y-C, Hsu C-P, Kao Y-H, Lin Y-K, Lin Y-J, et al. Electrophysiological characteristics of complex fractionated electrograms and high frequency activity in atrial fibrillation. Int $\mathrm{J}$ Cardiol [Internet] 2013 [cited 2014 Nov 3];168(3):2289-99. Available from: http://www.ncbi.nlm.nih.gov/pubmed/23465221
[13] Ganesan AN, Kuklik P, Lau DH, Brooks AG, Baumert M, Lim WW, et al. Bipolar Electrogram Shannon Entropy at Sites of Rotational Activation: Implications for Ablation of Atrial Fibrillation. Circ Arrhythmia Electrophysiol 2013;6(1):48-57.

[14] Ugarte JP. , Tobón C, Orozco-Duque A, Becerra MA, Bustamante J. Effect of the electrograms density in detecting and ablating the tip of the rotor during chronic atrial fibrillation: an in silico study. EP Europace 2015; 17 (Issue suppl_2): ii97-ii104

[15] Verma A, Jiang C, Betts T, Chen J, Deisenhofer I, Mantovan R, et al. Approaches to Catheter Ablation for Persistent Atrial Fibrillation. N Engl J Med 2015;372:1812-22.

[16] Berenfeld O, J. Jalife. Complex Fractionated Atrail Electrograms Is this the Beast to Tame in AF. Circ Arrhythm Electrophysiol 2011;4(4):426-8.

[17] Almeida TP, Jr JLS, Chu GS, Ng GA, Schlindwein FS, Sciences C, et al. Different Definitions of Complex Fractionated Atrial Electrograms do not Concur with the Clinical Perspective. Comput Cardiol (2010) 2013;40:1055-8.

[18] Ciaccio EJ, Biviano AB, Whang W, Garan H. Identification of Recurring Patterns in Fractionated Atrial Electrograms using New Transform Coefficients. Biomed Eng Online [Internet] 2012 [cited 2012 May 4];11(1):4. Available from: $h t t p: / / w w w . b i o m e d i c a l-e n g i-$ neering-online.com/content/11/1/4

[19] Eitel C, Hindricks G, Dagres N, Sommer P, Piorkowski C. EnSite Velocity cardiac mapping system: a new platform for 3D mapping of cardiac arrhythmias. Expert Rev Med Devices [Internet] 2010;7(2):185-92. Available from: http://www.ncbi.nlm.nih.gov/ pubmed/20214424

[20] Rolf S, Hindricks G, Sommer P, Richter S, Arya A, Kosiuk J, et al. Electroanatomical Mapping Of Atrial Fibrillation : Review Of The Current Techniques And Advances. J Atr Fibrillation 2015;7(4).

[21] Haissaguerre M, Jais P, Shah DC, Takahashi A, Hocini M, Quiniou $\mathrm{G}$, et al. Spontaneous Initiation of Atrial Fibrillation by Ectopic Beats Originating in the Pulmonary Veins. N Engl J Med 1998;339(10):659-66.

[22] Jalife J, Berenfeld O, Mansour M. Mother rotors and fibrillatory conduction: A mechanism of atrial fibrillation. Cardiovasc. Res.2002;54(2):204-16.

[23] Kalifa J, Tanaka K, Zaitsev A V, Warren M, Vaidyanathan R, Auerbach D, et al. Mechanisms of wave fractionation at boundaries of high-frequency excitation in the posterior left atrium of the isolated sheep heart during atrial fibrillation. Circulation 2006;113(5):626-33.

[24] Umapathy K, Masse S, Kolodziejska K, Veenhuyzen GD, Chauhan VS, Husain M, et al. Electrogram fractionation in murine HL-1 atrial monolayer model. Heart Rhythm 2008;5(7):1029-35.

[25] Narayan SM, Patel J, Mulpuru S, Krummen DE. Focal impulse and rotor modulation ablation of sustaining rotors abruptly terminates persistent atrial fibrillation to sinus rhythm with elimination on follow-up: A video case study. Hear Rhythm 2012;9(9):1436-9.

[26] Allessie MA, De Groot NMS, Houben RPM, Schotten U, Boersma E, Smeets JL, et al. Electropathological substrate of long-standing persistent atrial fibrillation in patients with structural heart disease longitudinal dissociation. Circ Arrhythmia Electrophysiol 2010;3(6):606-15. 
[27] Lee G, Kumar S, Teh A, Madry A, Spence S, Larobina M, et al. Epicardial wave mapping in human long-lasting persistent atrial fibrillation: Transient rotational circuits, complex wavefronts, and disorganized activity. Eur Heart J 2014;35(2):86-97.

[26] van Marion MSc DMS, Lanters Md EAH, Wiersma MSc M, Allessie 28 PhD MA, Brundel PhD BBJJM, de Groot Md PhD NMS. Diagnosis and Therapy of Atrial Fibrillation: The Past, The Present and The Future. J Atr Fibrillation 2015;8(2):1216.

[29] Allessie M, de Groot N. CrossTalk opposing view: Rotors have not been demonstrated to be the drivers of atrial fibrillation. J Physiol 2014;592(15):3167-70.

[30] Clarnette JA, Brooks AG, Mahajan R, et al. Outcomes of persistent and long-standing persistent atrial fibrillation ablation: a systematic review and meta-analysis. EP Eur [Internet]

2017;(February):1-11. Available from: https://goo.gl/mnZMZ2

[31] Nademanee K, McKenzie J, Kosar E, Schwab M, Sunsaneewitayakul B, Vasavakul T, et al. A new approach for catheter ablation of atrial fibrillation: Mapping of the electrophysiologic substrate. J Am Coll Cardiol 2004;43(11):2044-53.

[32] Oral H, Chugh A, Good E, Wimmer A, Dey S, Gadeela N, et al. Radiofrequency catheter ablation of chronic atrial fibrillation guided by complex electrograms. Circulation 2007;115(20):2606-12.

[33] Chen J, Lin Y, Chen L, Yu J, Du Z, Li S, et al. A decade of complex fractionated electrograms catheter-based ablation for atrial fibrillation: Literature analysis, meta-analysis and systematic review. IJC Hear Vessel 2014;4(1):63-72.

[34] Narayan SM, Shivkumar K, Krummen DE, Miller JM, Rappel WJ. Panoramic electrophysiological mapping but not electrogram morphology identifies stable sources for human atrial fibrillation: Stable atrial fibrillation rotors and focal sources relate poorly to fractionated electrograms. Circ Arrhythmia Electrophysiol 2013;6(1):58-67.

[35] Verma A, Jiang C, Betts TR, Chen J, Deisenhofer I, Mantovan R, et al. Approaches to Catheter Ablation for Persistent Atrial Fibrillation. N Engl J Med 2015;372(19):1812-22.

[36] Kirchhof P, Calkins H. Clinical update Catheter ablation in patients with persistent atrial fibrillation. Eur Heart J 2017;38:20-6.

[37] Reddy VY. Atrial Fibrillation: Unanswered Questions and Future Directions. Cardiol Clin [Internet] 2009 [cited 2012 May 8];27(1):201-16. Available from: http://linkinghub.elsevier.com/ retrieve/pii/S0733865108001069

[38] Elayi CS, Verma A, Di Biase L, Ching CK, Patel D, Barrett C, et al. Ablation for longstanding permanent atrial fibrillation: Results from a randomized study comparing three different strategies. Hear Rhythm 2008;5(12):1658-64.

[39] Magnani S, Muser D, Chik W, Santangeli P. Adjunct ablation strategies for persistent atrial fibrillation- beyond pulmonary vein isolation. J. Thorac. Dis.2015;7(2):178-84.

[40] Chen J, Lin Y, Chen L, Yu J, Du Z, Li S, et al. A decade of complex fractionated electrograms catheter-based ablation for atrial fibrillation: Literature analysis, meta-analysis and systematic review. IJC Hear Vessel [Internet] 2014 [cited 2014 Nov 3];1-10. Available from: http://linkinghub.elsevier.com/retrieve/pii/S2214763214000522

[41] Oketani N, Seitz J, Salazar M, Pisapia A. Ablation of complex fractionated electrograms is useful for catheter ablation of persistent atrial fi brillation : Protagonist point of view. Hear Rhythm [Internet] 2016;13(10):2098-100. Available from: http://dx.doi. org/10.1016/j.hrthm.2016.06.036
[42] Jadidi AS, Lehrmann H, Keyl C, Sorrel J, Markstein V, Minners J, et al. Ablation of Persistent Atrial Fibrillation Targeting Low-Voltage Areas With Selective Activation Characteristics. Circ Arrhythm Electrophysiol 2016;9(3):e002962.

[43] Vogler J, Willems S, Sultan A, Schreiber D, Lüker J, Servatius H, et al. Pulmonary Vein Isolation Versus Defragmentation the CHASE-AF Clinical Trial. J Am Coll Cardiol 2015;66(24):2743-52.

[44] Narayan SM, Baykaner T, Clopton P, Schricker A, Lalani GG, Krummen DE, et al. Ablation of rotor and focal sources reduces late recurrence of atrial fibrillation compared with trigger ablation alone: Extended follow-up of the CONFIRM trial (conventional ablation for atrial fibrillation with or without focal impulse and rotor modulat. J Am Coll Cardiol 2014;63(17):1761-8.

[45] Narayan SM, Krummen DE, Shivkumar K, Clopton P, Rappel W-J, Miller JM. Treatment of Atrial Fibrillation by the Ablation of Localized Sources. J Am Coll Cardiol [Internet] 2012;60(7):628-36.

[46] Haissaguerre M, Hocini M, Denis A, Shah AJ, Komatsu Y, Yamashita $\mathrm{S}$, et al. Driver domains in persistent atrial fibrillation. Circulation 2014;130(7):530-8.

[47] Buch E, Share M, Tung R, Benharash P, Sharma P, Koneru J, et al. Long-term clinical outcomes of focal impulse and rotor modulation for treatment of atrial fibrillation: A multicenter experience. Hear Rhythm 2016;13(3):636-41.

[48] Ravelli F, Masè M, Cristoforetti A, Marini M, Disertori M. The logical operator map identifies novel candidate markers for critical sites in patients with atrial fibrillation. Prog Biophys Mol Biol 2014;115(2-3):186-97.

[49] Sanders P, Nalliah CJ, Dubois R, Takahashi Y, Hocini M, Rotter M, et al. Frequency mapping of the pulmonary veins in paroxysmal versus permanent atrial fibrillation. J Cardiovasc Electrophysiol 2006;17(9):965-72.

[50] Novák D, Kremen V, Cuesta D, Schmidt K, Chudácek V, Lhotská L. Discrimination of endocardial electrogram disorganization using a signal regularity analysis. Conf Proc IEEE Eng Med Biol Soc [Internet] 2009;2009:1812-5. Available from: http://www.ncbi.nlm. nih.gov/pubmed/19963768

[51] Jalife J. Rotors and spiral waves in atrial fibrillation. J Cardiovasc Electrophysiol 2003;14(7):776-80.

[52] Botteron GW, Smith JM. A Technique for Measurement of the Extent of Spatial Organization of Atrial Activation During Atrial Fibrillation in the Intact Human Heart. IEEE Trans Biomed Eng 1995;42(6):579-86.

[53] Sanders P, Berenfeld O, Hocini M, Jais P, Vaidyanathan R, Hsu LF, et al. Spectral analysis identifies sites of high-frequency activity maintaining atrial fibrillation in humans. Circulation 2005;112(6):789-97.

[54] Everett IV TH, Kok LC, Vaughn RH, Moorman JR, Haines DE. Frequency domain algorithm for quantifying atrial fibrillation organization to increase defibrillation efficacy. IEEE Trans Biomed Eng 2001;48(9):969-78.

[55] Ng J, Kadish AH, Goldberger JJ. Technical considerations for dominant frequency analysis. J Cardiovasc Electrophysiol 2007;18(7):757-64.

[56] Elvan A, Linnenbank AC, Van Bemmel MW, Misier ARR, Delnoy PPHM, Beukema WP, et al. Dominant frequency of atrial fibrillation correlates poorly with atrial fibrillation cycle length. Circ Arrhythmia Electrophysiol 2009;2(6):634-44. 
[57] Houben RPM, de Groot NMS, Allessie M a. Analysis of fractionated atrial fibrillation electrograms by wavelet decomposition. IEEE Trans Biomed Eng [Internet] 2010;57(6):1388-98. Available from: http://www.ncbi.nlm.nih.gov/pubmed/20142164

[58] Kremen V, Lhotská L, Macas M, Cihák R, Vancura V, Kautzner J, et al. A new approach to automated assessment of fractionation of endocardial electrograms during atrial fibrillation. Physiol Meas [Internet] 2008 [cited $2014 \mathrm{Feb} 26$ ];29(12):1371-81. Available from: http://www.ncbi.nlm.nih.gov/pubmed/18946155

[59] Ng J, Borodyanskiy AI, Chang ET, Villuendas R, Dibs S, Kadish AH, et al. Measuring the complexity of atrial fibrillation electrograms. J Cardiovasc Electrophysiol 2010;21(6):649-55.

[60] Navoret N, Jacquir S, Laurent G, Binczak S. Detection of complex fractionated atrial electrograms using recurrence quantification analysis. IEEE Trans Biomed Eng 2013;60(7):1975-82.

[61] Ganesan AN, Kuklik P, Lau DH, Brooks AG, Baumert M, Lim WW, et al. Bipolar electrogram Shannon entropy at sites of rotational activation implications for ablation of atrial fibrillation. Circ Arrhythmia Electrophysiol 2013;6(1):48-57.

[62] Ugarte J, Orozco-Duque A, Tobón C, Kremen V, Novak D, Saiz J, et al. Dynamic approximate entropy electroanatomic maps detect rotors in a simulated atrial fibrillation model. PLoS One 2014;9(12):e114577.

[63] Cuesta-frau AD, Cirugeda-roldan E, Pico AM, Novak D, María E, Roldán C, et al. Atrial Electrogram Complex Fractionated Entropy Study. Exp Clin Cardiol 2014;20(9):5566-74.

[64] Orozco-Duque A, Novak D, Kremen V, Bustamante J. Multifractal analysis for grading complex fractionated electrograms in atrial fibrillation. Physiol Meas [Internet] 2015;36(11):2269-84. Available from: http://stacks.iop.org/0967-3334/36/i=11/a=2269?key=crossref. e7d25ed6d37af12d3473767bace5c3ea

[65] Urda-Benitez RD, Castro-Ospina A, Orozco-Duque A. Feature extraction based on time- singularity multifractal spectrum distribution in intracardiac atrial fibrillation signals Distribución tiempo singularidad del. TecnolLgicas 2017;20(40):97-111.
[66] Kremen V. Automated assessment of endocardial electrograms fractionation in human. 2008;

[67] Schilling C, Keller M, Scherr D, Oesterlein T, Haïssaguerre M, Schmitt C, et al. Fuzzy decision tree to classify complex fractionated atrial electrograms. Biomed Tech (Berl) [Internet] 2015 [cited 2015 Mar 26]; Available from: http://www.ncbi.nlm.nih.gov/ pubmed/25781659

[68] Wells J, Karp R, Kouchoukos N, Maclean W, James T, Waldo A. Characterization of Atrial Fibrillation in Man: Studies Following Open Heart Surgery. Pacing Clin Electrophysiol 1978;1(4):426-38.

[69] Schilling C, Keller M, Scherr D, Oesterlein T, Haïssaguerre M, Schmitt C, et al. Fuzzy decision tree to classify complex fractionated atrial electrograms. Biomed Tech 2015;60(3):245-55.

[70] Křemen V, Lhotská L, MacAš M, Čihák R, Vančura V, Kautzner J, et al. A new approach to automated assessment of fractionation of endocardial electrograms during atrial fibrillation. Physiol Meas 2008;29(12):1371-81.

[71] Hunter RJ, Diab I, Thomas G, Duncan E, Abrams D, Dhinoja M, et al. Validation of a classification system to grade fractionation in atrial fibrillation and correlation with automated detection systems. Europace 2009;11(12):1587-96.

[72] Nollo G, Marconcini M, Faes L, Bovolo F, Ravelli F, Bruzzone L. An automatic system for the analysis and classification of human atrial fibrillation patterns from intracardiac electrograms. IEEE Trans Biomed Eng 2008;55(9):2275-85.

[73] Duque SI, Orozco-Duque A, Kremen V, Novak D, Tobón C, Bustamante J. Feature subset selection and classification of intracardiac electrograms during atrial fibrillation. Biomed Signal Process Control [Internet] 2017;38:182-90. Available from: http:// dx.doi.org/10.1016/j.bspc.2017.06.005

[74] Orozco-Duque A, Bustamante J, Castellanos-Dominguez G. Semisupervised clustering of fractionated electrograms for electroanatomical atrial mapping. Biomed Eng Online 2016;15(1):44. 